\title{
TRACE FUNCTIONS AND GALOIS INVARIANT $p$-ADIC MEASURES
}

\author{
Marian VÂJÂItu AND Alexandru Zaharescu
}

\begin{abstract}
Let $p$ be a prime number, $\mathbb{Q}_{p}$ the field of $p$-adic numbers, $\overline{\mathbb{Q}}_{p}$ a fixed algebraic closure of $\mathbb{Q}_{p}$, and $\mathbb{C}_{p}$ the completion of $\overline{\mathbb{Q}}_{p}$ with respect to the $p$-adic valuation. We study trace functions associated to $p$-adic measures defined on compact subsets of $\mathbb{C}_{p}$ which are invariant under the action of the Galois group $G=\operatorname{Gal}_{\text {cont }}\left(\mathbb{C}_{p} / \mathbb{Q}_{p}\right)$.
\end{abstract}

\section{Introduction}

Let $p$ be a prime number, $\mathbb{Q}_{p}$ the field of $p$-adic numbers, $\overline{\mathbb{Q}}_{p}$ a fixed algebraic closure of $\mathbb{Q}_{p}$, and $\mathbb{C}_{p}$ the completion of $\overline{\mathbb{Q}}_{p}$ with respect to the $p$-adic valuation. The notion of a trace function associated to an element $T$ from $\mathbb{C}_{p}$ was introduced and investigated in [APZ3]. If $T$ is algebraic over $\mathbb{Q}_{p}$, and $L$ is a finite field extension of $\mathbb{Q}_{p}$ contained in $\overline{\mathbb{Q}}_{p}$ such that $T$ lies in $L$, then the $p$-adic number

$$
\operatorname{Tr} T:=\frac{\operatorname{Tr}_{L / \mathbb{Q}_{p}}(T)}{\left[L: \mathbb{Q}_{p}\right]}
$$

depends on $T$ only, and not on $L$. The significance of $\operatorname{Tr} T$ is that of the average value of the conjugates of $T$ over $\mathbb{Q}_{p}$. This idea of taking the average value rather than the sum of conjugates, may also be applied, as shown in $[\mathbf{A P Z 3}]$, to a rich class of elements $T$ from $\mathbb{C}_{p}$ which are transcendental over $\mathbb{Q}_{p}$. Given an element $T$ of $\mathbb{C}_{p}$, one takes its Galois orbit $C(T)=\left\{\sigma(T): \sigma \in \operatorname{Gal}_{\text {cont }}\left(\mathbb{C}_{p} / \mathbb{Q}_{p}\right)\right\}$, which is a compact subset of $\mathbb{C}_{p}$, and one considers the $p$-adic Haar distribution $\pi_{T}$ defined on $C(T)$. Then, by analogy with the case when $T$ is algebraic and $\operatorname{Tr} T$ is given by the average value of the conjugates of $T$ over $\mathbb{Q}_{p}$, one defines $\operatorname{Tr} T$

2000 Mathematics Subject Classification. 11S99.

Key words. Trace functions, rigid analytic functions, $p$-adic measures.

This work was partially supported by the Program ceex 2005 of the Romanian Ministry of Education and Research. 
for a general $T \in \mathbb{C}_{p}$ by the equality

$$
\operatorname{Tr} T=\int_{C(T)} x d \pi_{T}(x),
$$

provided that the integral on the right side of (2) is well defined. This is the case, for example, when the distribution $\pi_{T}$ is bounded, that is, when $\pi_{T}$ is a measure. The integral is also well defined when $T$ is a Lipschitzian element, in the sense of [APZ3]. The trace function $F(T, z)$ is defined by

$$
F(T, z)=\int_{C(T)} \frac{1}{1-z x} d \pi_{T}(x),
$$

for all those $z \in \mathbb{C}_{p}$ for which the integral is well defined. In the present paper we introduce and study some natural generalizations of the above objects. Let $G$ denote the group of continuous automorphisms of $\mathbb{C}_{p}$ over $\mathbb{Q}_{p}$. A compact subset $M$ of $\mathbb{C}_{p}$ is $G$-invariant provided that $\sigma(x) \in M$ for any $x \in M$ and $\sigma \in G$. If $M$ is a $G$-invariant compact subset of $\mathbb{C}_{p}$ and $\mu$ is a distribution on $M$ with values in $\mathbb{Q}_{p}$, we say that $\mu$ is $G$-invariant if $\mu(B)=\mu(\sigma(B))$ for any ball $B$ and any $\sigma \in G$. By a probability measure (or distribution) we mean a measure (respectively distribution) $\mu$ on $M$ for which $\mu(M)=1$. If $M$ is a $G$-invariant compact subset of $\mathbb{C}_{p}$ and $\mu$ is a $G$-invariant probability distribution on $M$, we define the trace of $\mu$ by the formula

$$
\operatorname{Tr} \mu=\int_{M} x d \mu(x),
$$

provided that the integral on the right side of (4) is well defined. We further associate a trace function $F(\mu, z)$ to $\mu$ by letting

$$
F(\mu, z)=\int_{M} \frac{1}{1-z x} d \mu(x),
$$

for all $z$ in $\mathbb{C}_{p}$ for which the integral is well defined. This is an analytic object that embodies a significant amount of algebraic data. For instance, recall that by Galois theory in $\mathbb{C}_{p}$ (see $\left.[\mathbf{T}],[\mathbf{S}],[\mathbf{A}]\right)$, closed subgroups of $G$ are in one-to-one correspondence with the closed subfields of $\mathbb{C}_{p}$. If $E$ is a closed subfield of $\mathbb{C}_{p}$ on which the trace map $\operatorname{Tr}$ is defined and continuous, and if $T$ is a generating element of $E$ over $\mathbb{Q}_{p}$ (see $[\mathbf{I Z}],[\mathbf{A P Z 1}],[\mathbf{A P Z 2}])$, then the trace map on the entire field $E$ is determined by the Taylor series expansion $F(T, z)=\sum_{n=0}^{\infty} \operatorname{Tr} T^{n} z^{n}$. Naturally, if $M$ and $\mu$ are $G$-invariant, one would like to be able to use the action of the Galois group $G$ to express and compute the above integrals. We achieve this goal in Theorems 1 and 2 from Section 4 below. 


\section{Preliminaries}

Let $M$ be a compact subset of $\mathbb{C}_{p}$, and let $\Omega(M)$ be the set of all open compact subsets of $M$. By a distribution on $M$ we mean a map $\mu: \Omega(M) \rightarrow \mathbb{C}_{p}$ that is finitely additive. If the set $\{\mu(B): B \in \Omega(M)\}$ is bounded in $\mathbb{C}_{p}, \mu$ is said to be a measure. Denote by $G=G a l_{\text {cont }}\left(\mathbb{C}_{p} / \mathbb{Q}_{p}\right)$ the group of all continuous automorphisms of $\mathbb{C}_{p}$ over $\mathbb{Q}_{p}$. Any $T \in \mathbb{C}_{p}$ has a $G$-orbit $C(T)=\{\sigma(T): \sigma \in G\}$, which is a compact subset of $\mathbb{C}_{p}$. Denote by $N(T, \varepsilon)$ the number of open balls of $\mathbb{C}_{p}$ of radius $\varepsilon$, any two disjoint, which cover $C(T)$. If $\varepsilon<\varepsilon^{\prime}$ then $N\left(T, \varepsilon^{\prime}\right)$ divides $N(T, \varepsilon)$. An element $T \in \mathbb{C}_{p}$ is called Lipschitzian if $\lim _{\varepsilon \rightarrow 0} \frac{\varepsilon}{|N(T, \varepsilon)|}=0$, where | | stands for the $p$-adic absolute value of the integer $N(T, \varepsilon)$. A compact subset $M$ of $\mathbb{C}_{p}$ is $G$-invariant provided that $\sigma(x) \in M$ for any $x \in M$ and $\sigma \in G$. If $M$ is a $G$-invariant compact subset of $\mathbb{C}_{p}$, given a distribution $\mu: \Omega(M) \rightarrow \mathbb{C}_{p}$, we say that $\mu$ is $G$-invariant if $\mu(D)=\mu(\sigma(D))$ for any $D \in \Omega(M)$ and any $\sigma \in G$. If $\mu(M)=1$ we call $\mu$ a probability distribution on $M$. For any $T \in \mathbb{C}_{p}$, the $p$-adic Haar distribution $\pi_{T}: \Omega(C(T)) \rightarrow \mathbb{Q}_{p}$ is the unique probability distribution on $C(T)$ with values in $\mathbb{Q}_{p}$ which is $G$-invariant. For more details and more general types of $p$-adic spaces and measures see $[\mathbf{M a n}],[\mathbf{K a}],[\mathbf{K o}]$, [Vi]. Any continuous function $f: M \rightarrow \mathbb{C}_{p}$ is integrable with respect to any measure $\mu$ on $M$, where the notion of integrability is defined as usual in terms of Riemann sums (see [Ko] the case $M=C(T)$, with $T \in \mathbb{C}_{p}$ Lipschitzian, any Lipschitzian function $f: C(T) \rightarrow \mathbb{C}_{p}$, is integrable (see $[\mathbf{A P Z 3}]$ ).

\section{The case $M=\cup_{j=1}^{r} C\left(T_{j}\right)$}

In this section we briefly discuss the case when $M$ is a finite union of distinct Galois orbits, $M=C\left(T_{1}\right) \cup \cdots \cup C\left(T_{r}\right)$ say. Our first objective is to obtain a characterization of all the $G$-invariant probability distributions on $M$ with values in $\mathbb{Q}_{p}$ in terms of the Haar distributions $\pi_{T_{1}}, \ldots, \pi_{T_{r}}$ defined on the Galois orbits $C\left(T_{1}\right), \ldots, C\left(T_{r}\right)$. Let $\mu: \Omega(M) \rightarrow \mathbb{Q}_{p}$ be finitely additive, satisfying $\mu(M)=1$, and $G$-invariant. Write any $D \in \Omega(M)$ as $D=D_{1} \cup \cdots \cup D_{r}$, where $D_{j}=D \cap C\left(T_{j}\right)$. Clearly each $D_{j}$ belongs to $\Omega(M)$. Thus $\mu(D)=\sum_{j=1}^{r} \mu\left(D_{j}\right)$. Let $\alpha_{1}, \ldots, \alpha_{r} \in \mathbb{Q}_{p}$ be given by

$$
\mu\left(C\left(T_{j}\right)\right)=\alpha_{j}, \quad j=1, \ldots, r .
$$

Note that

$$
\alpha_{1}+\cdots+\alpha_{r}=1
$$


Also,

$$
\mu(D)=\sum_{j=1}^{r} \alpha_{j} \pi_{T_{j}}\left(D_{j}\right)=\sum_{j=1}^{r} \alpha_{j} \pi_{T_{j}}\left(D \cap C\left(T_{j}\right)\right)
$$

for any $D \in \Omega(M)$. This expresses $\mu$ in terms of $\pi_{T_{1}}, \ldots, \pi_{T_{r}}$. Conversely, for any $\alpha_{1}, \ldots, \alpha_{r}$ of $\mathbb{Q}_{p}$ satisfying (7), formula (8) defines a $G$-invariant probability distribution on $M$. If $\pi_{T_{1}}, \ldots, \pi_{T_{r}}$ are bounded, so is $\mu$. Then any continuous function $f: M \rightarrow \mathbb{C}_{p}$ is integrable with respect to $\mu$, and

$$
\int_{M} f d \mu=\sum_{j=1}^{r} \alpha_{j} \int_{C\left(T_{j}\right)} f d \pi_{T_{j}}
$$

The equality (9) also holds if $T_{1}, \ldots, T_{r}$ are Lipschitzian, provided $f$ is Lipschitzian. Applying (9) with $f(x)=x$ gives a formula for the trace of $\mu$. Similarly, (9) and (5) give an expression for the trace function associated to $\mu$. We collect the results in the following proposition.

Proposition 1. Let $M=\cup_{j=1}^{r} C\left(T_{j}\right)$ with $T_{1}, \ldots, T_{r}$ Lipschitzian elements of $\mathbb{C}_{p}$ for which the Galois orbits $C\left(T_{1}\right), \ldots, C\left(T_{r}\right)$ are distinct. Let $\mu$ be a G-invariant probability on $M$ with values in $\mathbb{Q}_{p}$, and let $\alpha_{1}, \ldots, \alpha_{r}$ be given by (6). Then (i) $\operatorname{Tr} \mu$ is well defined, and satisfies the equality

$$
\operatorname{Tr} \mu=\sum_{j=1}^{r} \alpha_{j} \operatorname{Tr} T_{j}
$$

(ii) The trace series $F(\mu, Z)$ is well defined, and is given by

$$
F(\mu, Z)=\sum_{j=1}^{r} \alpha_{j} F\left(T_{j}, Z\right) .
$$

As a consequence, it follows by [APZ3] that under the hypothesis from Proposition 1, the function $z \mapsto F(\mu, z)$ is well defined and rigid analytic on $\mathbb{P}^{1}\left(\mathbb{C}_{p}\right) \backslash\left\{u \in \mathbb{P}^{1}\left(\mathbb{C}_{p}\right): \frac{1}{u} \in M\right\}$ (see also $[\mathbf{E}],[\mathbf{K o}],[\mathbf{K r}],[\mathbf{B}]$ ). This is its maximal domain of rigid analyticity if $\alpha_{1}, \ldots, \alpha_{r}$ are nonzero. Returning to (9), we may view its right side as an iterated integral, if we introduce an appropriate measure on the finite set $\mathcal{T}=\left\{T_{1}, \ldots, T_{r}\right\}$. Specifically, for any $T \in \mathbb{C}_{p}$, denote by $\nu_{T}$ the normalized Dirac measure on $\mathbb{C}_{p}$ concentrated at $T$. Next, with $\alpha_{1}, \ldots, \alpha_{r}$ given by (6), consider the measure $\nu$ on $\mathcal{T}$ defined as the linear combination

$$
\nu=\sum_{j=1}^{r} \alpha_{j} \nu_{T_{j}}
$$


Thus $\int_{\mathcal{T}} g d \nu=\sum_{j=1}^{r} \alpha_{j} g\left(T_{j}\right)$ for any $g: \mathcal{T} \rightarrow \mathbb{C}_{p}$. If now $f$ and $g$ are related by $g(T)=\int_{C(T)} f(y) d \pi_{T}(y)$, then $\int_{\mathcal{T}} g(T) d \nu(T)=\sum_{j=1}^{r} \alpha_{j} g\left(T_{j}\right)$, provided the two sides are well defined. We deduce that

$$
\int_{\mathcal{T}} \int_{C(T)} f(y) d \pi_{T}(y) d \nu(T)=\sum_{j=1}^{r} \alpha_{j} \int_{C\left(T_{j}\right)} f(y) d \pi_{T_{j}}(y),
$$

which, to simplify the notation, we also write as

$$
\int_{\mathcal{T}} \int_{C(T)} f d \pi_{T} d \nu=\sum_{j=1}^{r} \alpha_{j} \int_{C\left(T_{j}\right)} f d \pi_{T_{j}}
$$

Applying (12) to the function $f$ from (9) gives $\int_{M} f d \mu$ as an iterated integral. We state the result in the following proposition.

Proposition 2. Let $M=\cup_{j=1}^{r} C\left(T_{j}\right)$ with $T_{1}, \ldots, T_{r}$ Lipschitzian elements of $\mathbb{C}_{p}$ for which the Galois orbits $C\left(T_{1}\right), \ldots, C\left(T_{r}\right)$ are distinct. Let $\mu$ be a $G$-invariant probability distribution on $M$ with values in $\mathbb{Q}_{p}$, and define the measure $\nu$ on $\mathcal{T}=\left\{T_{1}, \ldots, T_{r}\right\}$ by (10) and (6). Then, for any Lipschitzian function $f: M \rightarrow \mathbb{C}_{p}$,

$$
\int_{M} f d \mu=\int_{\mathcal{T}} \int_{C(T)} f d \pi_{T} d \nu
$$

Corollary 1. Let $M, \mu, \mathcal{T}$ and $\nu$ be as in Proposition 2. Then

$$
\text { (i) } \quad \operatorname{Tr} \mu=\int_{\mathcal{T}} \operatorname{Tr} \operatorname{Td} \nu(T),
$$

and

$$
\text { (ii) } F(\mu, z)=\int_{\mathcal{T}} F(T, z) d \nu(T),
$$

for any $z \in \mathbb{P}^{1}\left(\mathbb{C}_{p}\right)$ for which both sides of (14) are well defined.

\section{The general case}

Proposition 2 above expresses the integral $\int_{M} f d \mu$ as an iterated integral. We achieved this result by an ad-hoc construction, which made use of a set $\mathcal{T}$ that is not intrinsically needed in our problem. We took advantage of the initial appearance of the elements $T_{1}, \ldots, T_{r}$ in the definition of the set $M$, but they do not have any special significance to the problem, and the set $\mathcal{T}$ may be replaced by any set of the form $\left\{\sigma_{1}\left(T_{1}\right), \ldots, \sigma_{r}\left(T_{r}\right)\right\}$, with $\sigma_{1}, \ldots, \sigma_{r} \in G$. Thus, rather than to insist that $\mathcal{T}$ be a subset of $\mathbb{C}_{p}$, it is more natural to define this set to be a set 
of Galois orbits. On $\mathbb{C}_{p}$ we have a natural equivalence relation: two elements $T_{1}$ and $T_{2}$ of $\mathbb{C}_{p}$ are equivalent if and only if there exists $\sigma \in G$ such that $T_{2}=\sigma\left(T_{1}\right)$. Denote by $\widehat{\mathbb{C}}_{p}$ the set of equivalence classes. Thus an element $t$ of $\widehat{\mathbb{C}}_{p}$ is a Galois orbit. Let $\Psi: \mathbb{C}_{p} \rightarrow \widehat{\mathbb{C}}_{p}$ the canonical map, which sends each element of $\mathbb{C}_{p}$ to its Galois orbit. On $\widehat{\mathbb{C}}_{p}$ we introduce a distance function $d$, by $d\left(t_{1}, t_{2}\right)=\inf \left\{|x-y|: x \in \Psi^{-1}\left(t_{1}\right), y \in \Psi^{-1}\left(t_{2}\right)\right\}$. We state some of the basic properties of $\widehat{\mathbb{C}}_{p}$ and its compact opens in the following lemma.

Lemma 1. With the above notations, $\widehat{\mathbb{C}}_{p}$ is a complete ultrametric space, and the map $\Psi: \mathbb{C}_{p} \rightarrow \widehat{\mathbb{C}}_{p}$ is 1-Lipschitzian. Moreover, for any $D \in \Omega(M)$ and $E \in \Omega(\widehat{M})$, we have $\Psi(D) \in \Omega(\widehat{M})$ and $\Psi^{-1}(E) \in \Omega(M)$.

To any $G$-invariant probability distribution $\mu$ on $M$ with values in $\mathbb{Q}_{p}$ we now associate a probability distribution $\widehat{\mu}$ on $\widehat{M}$ by $\widehat{\mu}(E)=\mu\left(\Psi^{-1}(E)\right)$, for any $E \in \Omega(\widehat{M})$. If $E \in \Omega(\widehat{M})$ is written as a disjoint union $E=$ $E_{1} \cup E_{2} \cup \cdots \cup E_{m}$, with $E_{1}, \ldots, E_{m} \in \Omega(\widehat{M})$, then $\widehat{\mu}(E)=\mu\left(\Psi^{-1}(E)\right)=$ $\sum_{i=1}^{m} \mu\left(\Psi^{-1}\left(E_{i}\right)\right)=\sum_{i=1}^{m} \widehat{\mu}\left(E_{i}\right)$, so $\widehat{\mu}$ is finitely additive. Also, $\widehat{\mu}(\widehat{M})=$ $\mu(M)=1$, so $\widehat{\mu}$ is a probability distribution on $\widehat{M}$. We claim that the map from the set of $G$-invariant probability distributions on $M$ with values in $\mathbb{Q}_{p}$ to the set of probability distributions on $\widehat{M}$ with values in $\mathbb{Q}_{p}$, given by $\mu \mapsto \widehat{\mu}$, is bijective. We first show that this map is injective. Assume that $\mu_{1} \neq \mu_{2}$ and $\widehat{\mu}_{1}=\widehat{\mu}_{2}$. Choose $D$ in $\Omega(M)$ for which $\mu_{1}(D) \neq \mu_{2}(D)$. Write $D$ as a disjoint union of closed balls in $M, D=B_{1} \cup B_{2} \cup \cdots \cup B_{n}$. Then $\sum_{i=1}^{n} \mu_{1}\left(B_{i}\right)=\mu_{1}(D) \neq$ $\mu_{2}(D)=\sum_{i=1}^{n} \mu_{2}\left(B_{i}\right)$. Choose a $B_{i}$ for which $\mu_{1}\left(B_{i}\right) \neq \mu_{2}\left(B_{i}\right)$. One has $\mu_{1}\left(\Psi^{-1}\left(\Psi\left(B_{i}\right)\right)\right)=\widehat{\mu}_{1}\left(\Psi\left(B_{i}\right)\right)=\widehat{\mu}_{2}\left(\Psi\left(B_{i}\right)\right)=\mu_{2}\left(\Psi^{-1}\left(\Psi\left(B_{i}\right)\right)\right)$. Let $\sigma_{1}, \ldots, \sigma_{N} \in G$ be such that $\Psi^{-1}\left(\Psi\left(B_{i}\right)\right)=\cup_{j=1}^{N} \sigma_{j}\left(B_{i}\right)$, with $\sigma_{j}\left(B_{i}\right)$ disjoint. Then $\sum_{j=1}^{N} \mu_{1}\left(\sigma_{j}\left(B_{i}\right)\right)=\mu_{1}\left(\Psi^{-1}\left(\Psi\left(B_{i}\right)\right)\right)=\mu_{2}\left(\Psi^{-1}\left(\Psi\left(B_{i}\right)\right)\right)=$ $\sum_{j=1}^{N} \mu_{2}\left(\sigma_{j}\left(B_{i}\right)\right)$. Since $\mu_{1}$ and $\mu_{2}$ are $G$-invariant, $\sum_{j=1}^{N} \mu_{1}\left(\sigma_{j}\left(B_{i}\right)\right)=$ $N \mu_{1}\left(B_{i}\right)$, and $\sum_{j=1}^{N} \mu_{2}\left(\sigma_{j}\left(B_{i}\right)\right)=N \mu_{2}\left(B_{i}\right)$. Hence $\mu_{1}\left(B_{i}\right)=\mu_{2}\left(B_{i}\right)$, and we obtain a contradiction. This shows that the map $\mu \mapsto \widehat{\mu}$ is injective. The surjectivity is proved by similar reasonings. We collect the results in the following theorem.

Theorem 1. Let $M$ be a G-invariant compact subset of $\mathbb{C}_{p}$. Then the $G$-invariant probability distributions on $M$ with values in $\mathbb{Q}_{p}$ are in oneto-one correspondence with the probability distributions on $\widehat{M}$ with values in $\mathbb{Q}_{p}$, via the map $\mu \mapsto \widehat{\mu}$. 
We note that $\widehat{\mu}$ may be bounded even if $\mu$ is not bounded. For instance, if $M$ is a finite union of Galois orbits, then $\widehat{\mu}$ is bounded, regardless of whether $\mu$ is bounded or not. Note also that even if $\mu$ and $\widehat{\mu}$ are both bounded, we might not be able to obtain (13). For instance, if $M=\cup_{j=1}^{r} C\left(T_{j}\right)$, with $\pi_{T_{j}}$ bounded for all $j$ with the exception of $j=1$, then any $G$-invariant probability distribution $\mu$ on $M$ with values in $\mathbb{Q}_{p}$ for which the corresponding $\alpha_{1}$ defined as in (6) vanishes, will be bounded. In that case the left side of (13) will be well defined, for any continuous function $f: M \rightarrow \mathbb{C}_{p}$. On the other hand, depending on $f$ and $\pi_{T_{1}}$, the right side of (13) might be undefined, as the inner integral in (13) may be undefined at the point $T=T_{1}$. In what follows we will avoid such situations by restricting to the case when $\widehat{\mu}$ and the Haar distributions $\pi_{T_{j}}$ are bounded.

We return to the more general case of a $G$-invariant compact subset $M$ of $\mathbb{C}_{p}$ and make the following assumption: there exists a positive real number $A$, depending on $M$, such that for any $T \in M$ and any $D \in \Omega(C(T))$, one has

$$
\left|\pi_{T}(D)\right| \leq A
$$

Here the absolute value on the left side of (15) is the $p$-adic absolute value on $\mathbb{Q}_{p}$. The condition, in other words, says that the Haar distributions $\pi_{T}$, with $T \in M$, are uniformly bounded. We remark that, although $M$ is compact, it is not enough to assume that each $\pi_{T}$ with $T$ in $M$ is bounded in order to conclude that these distributions are uniformly bounded. As an example, choose a sequence $\left(\alpha_{n}\right)_{n \in \mathbb{N}}$ of algebraic elements over $\mathbb{Q}_{p}$, which is convergent to an algebraic element $\alpha$, and such that the exponent of $p$ in the degree $\operatorname{deg} \alpha_{n}$ of $\alpha_{n}$ over $\mathbb{Q}_{p}$ tends to infinity as $n \rightarrow \infty$. Set $M=C(\alpha) \cup \cup_{n \in \mathbb{N}} C\left(\alpha_{n}\right)$. Then $M$ is compact and $G$ invariant. Also, $\pi_{\alpha}$ and $\pi_{\alpha_{n}}$ are bounded. On the other hand, for any point $U$ in $C\left(\alpha_{n}\right), \pi_{\alpha_{n}}(\{U\})=\frac{1}{\operatorname{deg} \alpha_{n}}$. Since the exponent of $p$ in $\operatorname{deg} \alpha_{n}$ tends to infinity as $n \rightarrow \infty$, the Haar distributions are not uniformly bounded.

We now return to the case of a general $G$-invariant compact set $M$ for which the Haar distributions $\pi_{T}$, with $T$ in $M$, are uniformly bounded. Let $\mu$ be a $G$-invariant probability distribution on $M$ for which $\widehat{\mu}$ is bounded. Then $\mu$ will also be bounded. We are interested to see whether an analogue of formula (13) still holds in this generality. For any $t \in \widehat{M}$ we denote by $C_{t}$ the Galois orbit in $\mathbb{C}_{p}$ which defines $t$, and by $\pi_{t}$ the Haar distribution on $C_{t}$. Let $f: M \rightarrow \mathbb{C}_{p}, f$ continuous. The analogue 
of (13) reads

$$
\int_{M} f d \mu=\int_{\widehat{M}} \int_{C_{t}} f d \pi_{t} d \widehat{\mu} .
$$

Since $f$ is continuous and $\mu$ is bounded, the left side of (16) is well defined. On the right side of (16), the outer integral, over $\widehat{M}$, is with respect to the variable $t$. For each fixed $t \in \widehat{M}$, the inner integral is well defined, as one integrates on $C_{t}$ the restriction of $f$, which is continuous with respect to $\pi_{t}$, which is bounded. In order to show that the right side of (16) is well defined, it will be enough to prove that the function defined on $\widehat{M}$ by $t \mapsto \int_{C_{t}} f d \pi_{t}$ is continuous. Then, since $\widehat{\mu}$ is bounded, the outer integral, and then also the entire right side of (16) will be well defined.

To show that the above function is continuous, fix $t_{0} \in \widehat{M}$. We need to show that $\int_{C_{t}} f d \pi_{t} \rightarrow \int_{C_{t_{0}}} f d \pi_{t_{0}}$, as $t \rightarrow t_{0}$. Fix $\varepsilon>0$. Since $f$ is continuous and $M$ compact, from uniform continuity, there is a $\delta>0$ such that, for any $x, y \in M$ with $|y-x| \leq \delta$, one has $|f(y)-f(x)| \leq \varepsilon$. Let $t$ in $\widehat{M}$ with $d\left(t, t_{0}\right)<\delta$. Write $M$ as a disjoint union of closed balls of radius $\delta$. Some of them intersect $C_{t_{0}}$. Denote these balls by $B_{1}, B_{2}, \ldots, B_{N}$. Since $d\left(t, t_{0}\right)<\delta$, each ball $B_{j}$, with $1 \leq j \leq N$, has a nonempty intersection with $C_{t}$. Let $x_{j} \in C_{t_{0}} \cap B_{j}$ and $y_{j} \in C_{t} \cap B_{j}$, for $j=1,2, \ldots, N$.

Let $S_{1}=\sum_{j=1}^{N} f\left(x_{j}\right) \pi_{t_{0}}\left(C_{t_{0}} \cap B_{j}\right)$ and $S_{2}=\sum_{j=1}^{N} f\left(y_{j}\right) \pi_{t}\left(C_{t} \cap B_{j}\right)$. The sums $S_{1}$ and $S_{2}$ are Riemann sums for the integrals $\int_{C_{t_{0}}} f d \pi_{t_{0}}$ and respectively $\int_{C_{t}} f d \pi_{t}$. We need to show that these two integrals are close to each other, provided that $\varepsilon$ is small enough, and for this it is enough to show that $S_{1}$ and $S_{2}$ are close to these integrals, and that $S_{1}$ and $S_{2}$ are close to each other. The balls $B_{j}$ being conjugate, $\pi_{t_{0}}\left(C_{t_{0}} \cap B_{j}\right)=$ $\pi_{t}\left(C_{t} \cap B_{j}\right)=\frac{1}{N}$, hence $S_{1}=\frac{1}{N} \sum_{j=1}^{N} f\left(x_{j}\right)$, and $S_{2}=\frac{1}{N} \sum_{j=1}^{N} f\left(y_{j}\right)$. Therefore, $\left|S_{1}-S_{2}\right|=\left|\frac{1}{N} \sum_{j=1}^{N}\left(f\left(x_{j}\right)-f\left(y_{j}\right)\right)\right| \leq \frac{1}{|N|} \max _{1 \leq j \leq N} \mid f\left(x_{j}\right)-$ $f\left(y_{j}\right) \mid$. Here $x_{j}, y_{j} \in B_{j},\left|x_{j}-y_{j}\right| \leq \delta$, so $\left|f\left(x_{j}\right)-f\left(y_{j}\right)\right| \leq \varepsilon$, and consequently $\left|S_{1}-S_{2}\right| \leq \frac{\varepsilon}{|N|}$. By (15), the $p$-power in $N$ is bounded in terms of $M$ only. It follows that

$$
\left|S_{1}-S_{2}\right| \rightarrow 0, \quad \text { as } \quad \varepsilon \rightarrow 0 .
$$

Next, with $\varepsilon$ and $\delta$ fixed, take a small $\delta^{\prime}>0$ and write each closed ball $B_{j}$ of radius $\delta$ as a disjoint union of closed balls of radius $\delta^{\prime}, B_{j}=$ $\cup_{i=1}^{r} B_{j i}$. The balls $B_{j}$ being conjugate, each of them is a disjoint union of the same number $r$ of closed balls of radius $\delta^{\prime}$. For any $j \in\{1, \ldots, N\}$, 
out of the $r$ balls $B_{j i}, 1 \leq i \leq r$, the same number of them, $r^{\prime}$ say, have a nonempty intersection with $C_{t_{0}}$. After redenoting the balls if necessary, assume that the balls which intersect $C_{t_{0}}$ are $B_{j i}, 1 \leq i \leq r^{\prime}$, $1 \leq j \leq N$. Choose $x_{j i}$ in $B_{j i} \cap C_{t_{0}}$, and consider the Riemann sum $S_{1}^{\prime}=\sum_{j=1}^{N} \sum_{i=1}^{r^{\prime}} f\left(x_{j i}\right) \pi_{t_{0}}\left(B_{j i} \cap C_{t_{0}}\right)$.

Let $\delta^{\prime}$ be small enough so that for any choice of $x_{j i},\left|S_{1}^{\prime}-\int_{C_{t_{0}}} f d \pi_{t_{0}}\right|<\varepsilon$. The sets $B_{j i} \cap C_{t_{0}}$ are conjugate, and $S_{1}^{\prime}=\frac{1}{N r^{\prime}} \sum_{j=1}^{N} \sum_{i=1}^{r^{\prime}} f\left(x_{j i}\right)$. By our assumptions, $\left|\pi_{t_{0}}\left(B_{j i} \cap C_{t_{0}}\right)\right|=\left|\frac{1}{N r^{\prime}}\right|$ is bounded by a number which depends on $M$ only. Using the fact that $\left|f\left(x_{j}\right)-f\left(x_{j i}\right)\right| \leq \varepsilon$, since $x_{j}, x_{j i} \in B_{j}$ for all $i$ and $j$, we have $\left|S_{1}-S_{1}^{\prime}\right| \leq\left|\frac{1}{N r^{\prime}}\right| \max _{\substack{1 \leq N \leq r^{\prime} \\ 1 \leq i \leq r^{\prime}}} \mid f\left(x_{j}\right)-$ $f\left(x_{j i}\right)|\leq| \frac{1}{N r^{\prime}} \mid \varepsilon$, so $\left|S_{1}-S_{1}^{\prime}\right| \rightarrow 0$, as $\varepsilon \rightarrow 0$. We deduce that

$$
S_{1} \rightarrow \int_{C_{t_{0}}} f d \pi_{t_{0}}, \quad \text { as } \quad \varepsilon \rightarrow 0
$$

regardless of the choice of the parameters appearing in the definition of $S_{1}$. Similarly,

$$
\left|S_{2}-\int_{C_{t}} f d \pi_{t}\right| \rightarrow 0, \quad \text { as } \quad \varepsilon \rightarrow 0 .
$$

By (17), (18), and (19), we conclude that $\int_{C_{t}} f d \pi_{t} \rightarrow \int_{C_{t_{0}}} f d \pi_{t_{0}}$, as $t \rightarrow t_{0}$, which proves the continuity of the map $t \mapsto \int_{C_{t}} f d \pi_{t}$.

It remains to show that the two sides of formula (16) are equal. We first reduce to the case of step functions. Fix a continuous function $f: M \rightarrow \mathbb{C}_{p}$. By the uniform continuity of $f$, for any $\varepsilon>0$, there is a $\delta>0$ such that $|f(x)-f(y)| \leq \varepsilon$ for any $x, y \in M$ with $|x-y| \leq \delta$. Write $M$ as a disjoint union of closed balls of radius $\delta, M=\cup_{j=1}^{m} B_{j}$. Choose $z_{j}$ in $B_{j}$, and let $g=\sum_{j=1}^{m} f\left(z_{j}\right) \chi_{B_{j}}$, where $\chi_{B_{j}}$ denotes the characteristic function of the ball $B_{j}$. Denote $h=f-g$. Then $|h(z)|=|f(z)-g(z)| \leq \varepsilon$, for any $z \in M$. It follows that for any Riemann sum $S$ associated with the integral $\int_{M} h d \mu, S=\sum_{i=1}^{L} h\left(x_{i}\right) \mu\left(D_{i}\right)$, where $D_{1}, \ldots, D_{L} \in \Omega(M)$ form a partition of $M$, and $x_{i} \in D_{i}$ for $1 \leq i \leq L$, one has

$$
|S| \leq \max _{1 \leq i \leq L}\left|h\left(x_{i}\right) \mu\left(D_{i}\right)\right| \leq \varepsilon A(\mu)
$$

where $A(\mu)=\sup _{D \in \Omega(M)}|\mu(D)|<\infty$. By applying (20) to a sequence of Riemann sums converging to $\int_{M} h d \mu$, we obtain

$$
\left|\int_{M} h d \mu\right| \leq \varepsilon A(\mu) \text {. }
$$


Similarly, the sup norm of $h$ on $C_{t}$, with $t \in \widehat{M}$, is bounded by $\varepsilon$, and $\left|\int_{C_{t}} h d \pi_{t}\right| \leq \varepsilon A\left(\pi_{t}\right)$, where $A\left(\pi_{t}\right)=\sup _{D \in \Omega(M)}\left|\pi_{t}(D)\right|$. Define $H: \widehat{M} \rightarrow \mathbb{C}_{p}$ by $H(t):=\int_{C_{t}} h d \pi_{t}$. Then $\sup _{t \in \widehat{M}}|H(t)| \leq \varepsilon \sup _{t \in \widehat{M}} A\left(\pi_{t}\right)$. By our assumption $\sup _{t \in \widehat{M}} A\left(\pi_{t}\right)<\infty$. We find as before that

$$
\left|\int_{\widehat{M}} \int_{C_{t}} h d \pi_{t} d \widehat{\mu}\right|=\left|\int_{\widehat{M}} H(t) d \widehat{\mu}\right| \leq \varepsilon A(\widehat{\mu}) \sup _{t \in \widehat{M}} A\left(\pi_{t}\right),
$$

where $A(\widehat{\mu})=\sup _{D \in \Omega(\widehat{M})}|\widehat{\mu}(D)|<\infty$. Assuming that (16) holds for all the step functions defined on $M$, we have

$$
\int_{M} g d \mu=\int_{\widehat{M}} \int_{C_{t}} g d \pi_{t} d \widehat{\mu} .
$$

Combining (21), (22) and (23) we derive the inequality

$$
\left|\int_{M} f d \mu-\int_{\widehat{M}} \int_{C_{t}} f d \pi_{t} d \widehat{\mu}\right| \leq \varepsilon \max \left\{A(\mu), A(\widehat{\mu}) \sup _{t \in \bar{M}} A\left(\pi_{t}\right)\right\} .
$$

We now let $\varepsilon \rightarrow 0$ and conclude that formula (16) holds true for $f$. So we are done provided we show that formula (16) holds true for step functions. Clearly both sides of (16) are linear operators. Therefore it is enough to prove (16) in the particular case when $f$ is the characteristic functions of a closed ball $B$ in $M$. In this case, the left side of (16) reduces to

$$
\int_{M} \chi_{B} d \mu=\mu(B)
$$

while the right side of (16) equals

$$
\int_{\widehat{M}} \int_{C_{t}} \chi_{B} d \pi_{t} d \widehat{\mu}=\int_{\widehat{M}} \pi_{t}\left(B \cap C_{t}\right) d \widehat{\mu} .
$$

Let us denote $E=\Psi(B)$. We know that

$$
\widehat{\mu}(E)=\mu\left(\Psi^{-1}(E)\right)=\mu\left(\Psi^{-1}(\Psi(B))\right) .
$$

We also know that $\Psi^{-1}(\Psi(B))$ can be written as a finite disjoint union of closed balls of the form

$$
\Psi^{-1}(\Psi(B))=\cup_{i=1}^{N} \sigma_{i}(B),
$$

where $\sigma_{1}, \sigma_{2}, \ldots, \sigma_{N} \in G$. By (24), (26) and (27) we obtain

$$
\int_{M} \chi_{B} d \mu=\mu(B)=\frac{1}{N} \mu\left(\Psi^{-1}(\Psi(B))\right)=\frac{1}{N} \widehat{\mu}(E) .
$$


On the other hand, it is easy to see that $\pi_{t}\left(B \cap C_{t}\right)=\frac{1}{N}$ for any $t \in E$, while for $t \in \widehat{M} \backslash E$ one has $\pi_{t}\left(B \cap C_{t}\right)=0$. Combining this with (25) we find that

$$
\int_{\widehat{M}} \int_{C_{t}} \chi_{B} d \pi_{t} d \widehat{\mu}=\int_{\widehat{M}} \pi_{t}\left(B \cap C_{t}\right) d \widehat{\mu}=\int_{E} \frac{1}{N} d \widehat{\mu}=\frac{1}{N} \widehat{\mu}(E) .
$$

Finally, by (28) and (29) we see that formula (16) holds true for $\chi_{B}$. We collect the results in the following theorem.

Theorem 2. Let $M$ be a $G$-invariant compact subset of $\mathbb{C}_{p}$ and let $\mu$ be a $G$-invariant probability distribution on $M$ with values in $\mathbb{Q}_{p}$. Assume that $\widehat{\mu}$ is bounded, and that the Haar distributions $\pi_{t}$, with $t \in \widehat{M}$, are uniformly bounded. Then for any continuous function $f: M \rightarrow \mathbb{C}_{p}$,

$$
\int_{M} f d \mu=\int_{\widehat{M}} \int_{C_{t}} f d \pi_{t} d \widehat{\mu} \text {. }
$$

As a corollary we obtain an expression for the trace of $\mu$ and the trace function associated to $\mu$ in terms of the trace $\operatorname{Tr} t$ and respectively the trace function $F(t, z)$ associated to the Galois orbits $C_{t}$, with $t$ in $\widehat{M}$.

Corollary 2. Let $M$ and $\mu$ be as in the statement of Theorem 2. Then

$$
\text { (i) } \operatorname{Tr} \mu=\int_{\widehat{M}} \operatorname{Tr} t d \widehat{\mu},
$$

and

$$
\text { (ii) } F(\mu, z)=\int_{\widehat{M}} F(t, z) d \widehat{\mu},
$$

for any $z \in \mathbb{P}^{1}\left(\mathbb{C}_{p}\right)$ for which both sides of (30) are well defined.

Acknowledgement. The authors are grateful to the referee for many useful comments and suggestions.

\section{References}

[APZ1] V. Alexandru, N. Popescu and A. Zaharescu, On the closed subfields of $\mathbb{C}_{p}$, J. Number Theory 68(2) (1998), 131-150.

[APZ2] V. Alexandru, N. Popescu and A. Zaharescu, The generating degree of $\mathbb{C}_{p}$, Canad. Math. Bull. 44(1) (2001), 3-11. 
[APZ3] V. Alexandru, N. Popescu and A. Zaharescu, Trace on $\mathbb{C}_{p}$, J. Number Theory 88(1) (2001), 13-48.

[Ar] E. ARTin, "Algebraic numbers and algebraic functions", Gordon and Breach Science Publishers, New York-London-Paris, 1967.

[A] J. Ax, Zeros of polynomials over local fields-The Galois action, J. Algebra 15 (1970), 417-428.

[B] D. BARSky, Transformation de Cauchy p-adique et algèbre d'Iwasawa, Math. Ann. 232(3) (1978), 255-266.

[E] A. Escassut, Algèbres de Banach ultramètriques et algèbres de Krasner-Tate, in: "Prolongement analytique et algèbres de Banach ultramétriques", pp. 1-107, 219, Astérisque 10, Sociéte Mathématique de France, Paris (1973).

[IZ] A. IOviţă AND A. Zaharescu, Completions of r.a.t.-valued fields of rational functions, J. Number Theory 50(2) (1995), 202-205.

[Ka] N. M. Katz, The Eisenstein measure and $p$-adic interpolation, Amer. J. Math., 99(2) (1977), 238-311.

[Ko] N. KoBlitz, "p-adic analysis: a short course on recent work", London Mathematical Society Lecture Note Series 46, Cambridge University Press, Cambridge-New York, 1980.

[Kr] M. Krasner, Rapport sur le prolongement analytique dans les corps valués completes par la méthode des éléments analytiques quasi-connexes, in: "Table Ronde d'Analyse non archimédienne" (Paris, 1972), Bull. Soc. Math. France, Mém. 39-40, Soc. Math. France, Paris, 1974, pp. 131-254.

[Man] Ju. I. Manin, Periods of cusp forms, and $p$-adic Hecke series, (Russian), Mat. Sb. (N.S.) 92(134) (1973), 378-401, 503.

[MSD] B. Mazur and P. Swinnerton-Dyer, Arithmetic of Weil curves, Invent. Math. 25 (1974), 1-61.

[SCH] W. H. Schiкhof, "Ultrametric calculus", An introduction to p-adic analysis, Cambridge University Press, Cambridge, 1984.

[S] S. Sen, On automorphisms of local fields, Ann. of Math. (2) 90 (1969), 33-46.

[T] J. T. Tate, p-divisible groups, in: "Proc. Conf. Local Fields" (Driebergen, 1966), Springer, Berlin, 1967, pp. 158-183.

[Vi] M. M. VIŠIK, Nonarchimedean measures associated with Dirichlet series, (Russian), Mat. Sb. (N.S.) 99(141) (1976), no. 2, 248-260, 296. 
Trace Functions and Galois Invariant $p$-Adic Measures 55

Marian Vâjâitu:

Institute of Mathematics of the Romanian Academy

P. O. Box 1-764

Bucharest 70700

Romania

E-mail address: Marian.Vajaitu@imar.ro

Alexandru Zaharescu:

Department of Mathematics

University of Illinois at Urbana-Champaign

Altgeld Hall, 1409 W. Green Street

Urbana, IL, 61801

USA

E-mail address: zaharesc@math.uiuc.edu

Primera versió rebuda el 9 d'octubre de 2004,

darrera versió rebuda el 20 de juny de 2005. 